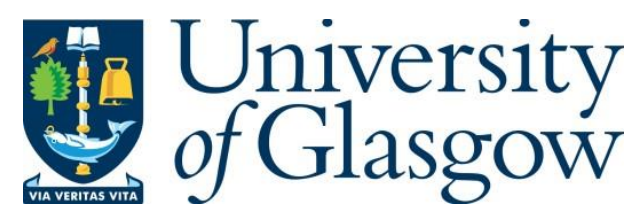

Anguita, A. (2018) Self-assessment: weight loss and anorexia in a Fox Terrier.

Companion Animal, 23(3), pp. 136-139.

There may be differences between this version and the published version. You are advised to consult the publisher's version if you wish to cite from it.

http://eprints.gla.ac.uk/158888/

Deposited on: 25 May 2018

Enlighten - Research publications by members of the University of Glasgow http://eprints.gla.ac.uk 


\title{
Self-assessment: weight loss and anorexia in a Fox Terrier
}

\author{
Antonio Anguita DVM MRCVS, Small Animal Intern, School of Veterinary \\ Medicine, University of Glasgow, Bearsden Road, Glasgow, G61 1QH
}

A ten-year-old male entire Fox Terrier was presented with abdominal swelling, weight loss and anorexia.

No previous health problems were reported. Progressive abdominal distention had first been noticed starting 2 months ago, accompanied by weight loss. The owner also reported that the dog had not been defecating during the last weeks but was still urinating, although urination was seen more frequently with smaller volumes being produced. Appetite was reduced and the dog had become polydipsic. No vomiting episodes had been reported at the time of presentation.

\section{Initial investigation}

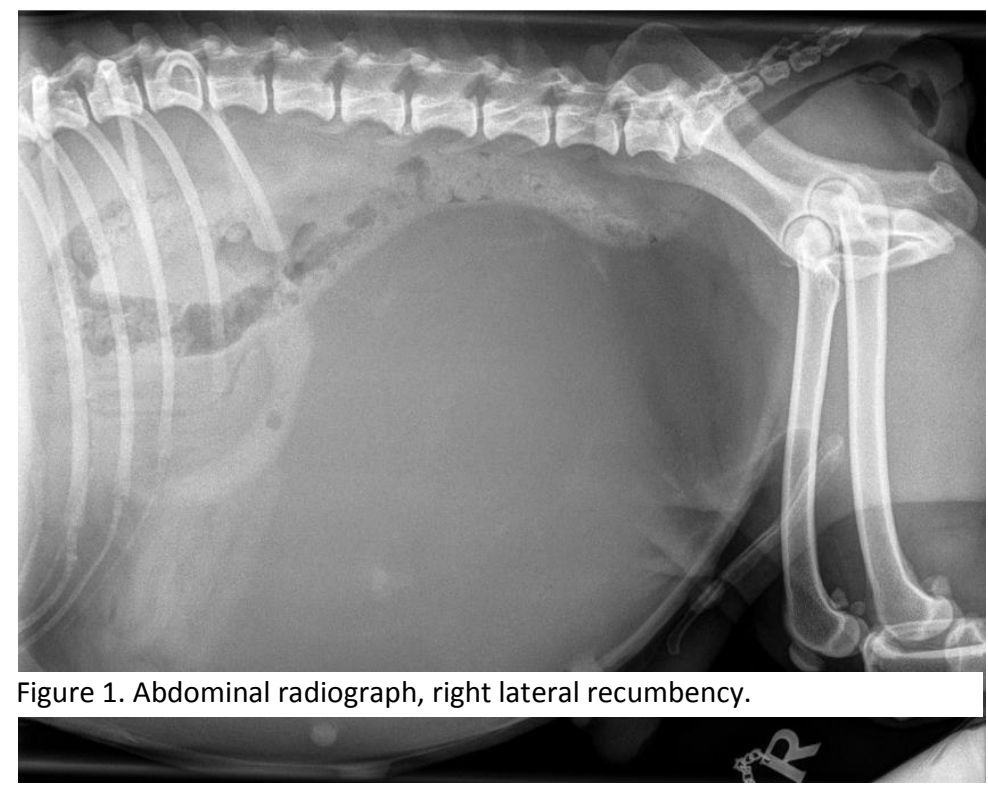

\section{Clinical examination}

On clinical examination the patient was bright, alert and responsive. Cardiovascular and respiratory systems were unremarkable. Abdominal palpation was limited as the abdomen was very distended. Lymph nodes were completely normal on palpation. The dog's nipples were subjectively considered to be larger and more prominent than is typical in male dogs. His right testicle was relatively soft on palpation and much larger than the left; this had been noticed several months before the other symptoms started to appear. Rectal palpation revealed an obstruction making it impossible to palpate further than 2 $\mathrm{cm}$ into the rectum.

\section{Radiography}

Using a Siemens MultixTop x-ray machine (with an AGFA CR35 CR system), thoracic (right lateral recumbency ( $R L R)$, left lateral recumbency (LLR), and dorsoventral (DV) and abdominal (ventrodorsal (VD) and right lateral

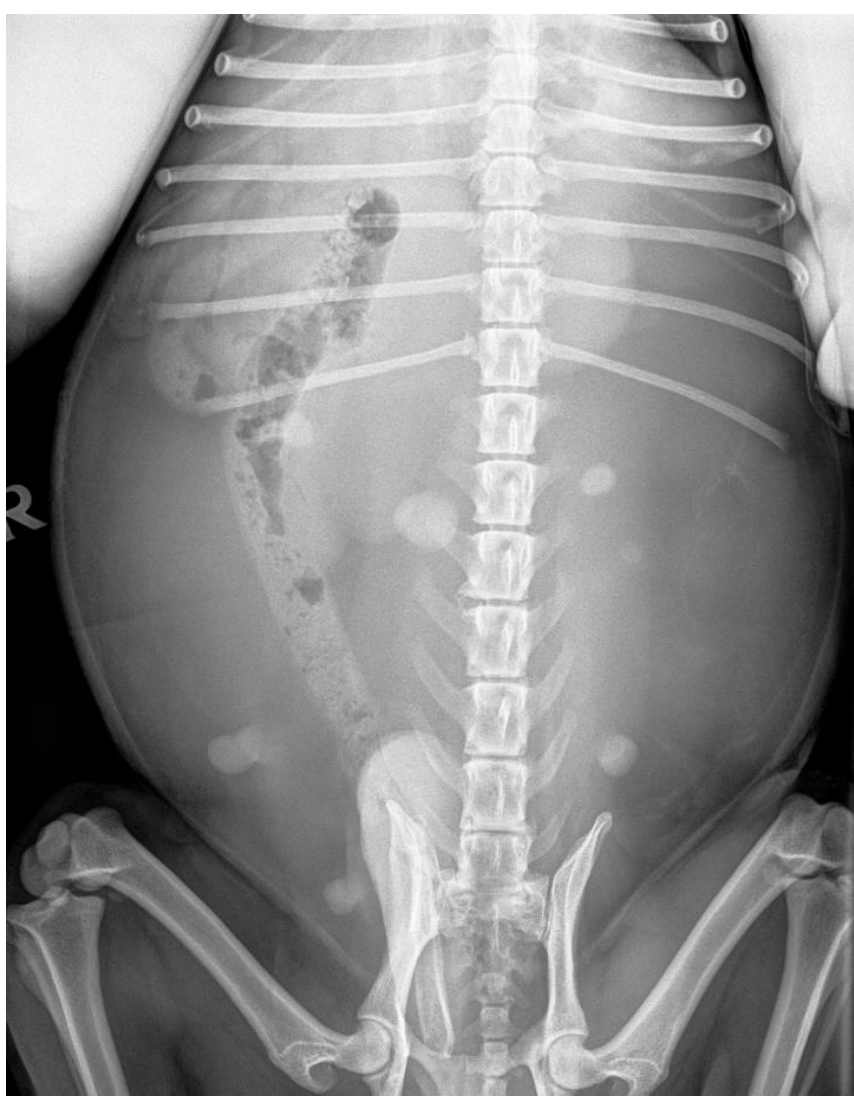

Figure 2. Abdominal radiograph, ventro-dorsal recumbency. 
recumbency (RLR)) radiographs were obtained under sedation using a combination of 10 ug of medetomidine and $1 \mathrm{mg}$ of butorphanol intravenously. Both abdominal projections are shown in Figures 1 and 2. A full abdominal ultrasound was also performed using a GE Logiq 9 Ultrasound machine ( $8 \mathrm{MHz}$ microconvex probe for general abdomen, $12 \mathrm{MHz}$ linear transducer for testes).

\section{What abnormalities can you see on the radiographs? Construct a differential diagnosis list based on your findings}

Collimation of the radiographs in the study was not perfectly adjusted as the diaphragm and the full extent of the liver are not included fully on the abdominal projections.

No obvious abnormalities are seen within the thoracic cavity. However, there is reduced pulmonary capacity bilaterally, with the diaphragm intact but pushed cranially to the level of the $6^{\text {th }}$ rib, associated with the abdominal changes. The heart size and position within the thorax are normal. A ventro-dorsal view of the thorax is shown in Figure 3.

Within the abdominal cavity, there is marked cranial displacement of the majority of the abdominal organs, with the descending colon displaced dorsally and to the right side of midline. No clear bladder shadow can be identified; it is presumably displaced into the pelvic canal, very reduced in size, displacing and reducing the size of the rectum as well. The majority of the abdominal cavity is occupied by fat opacity

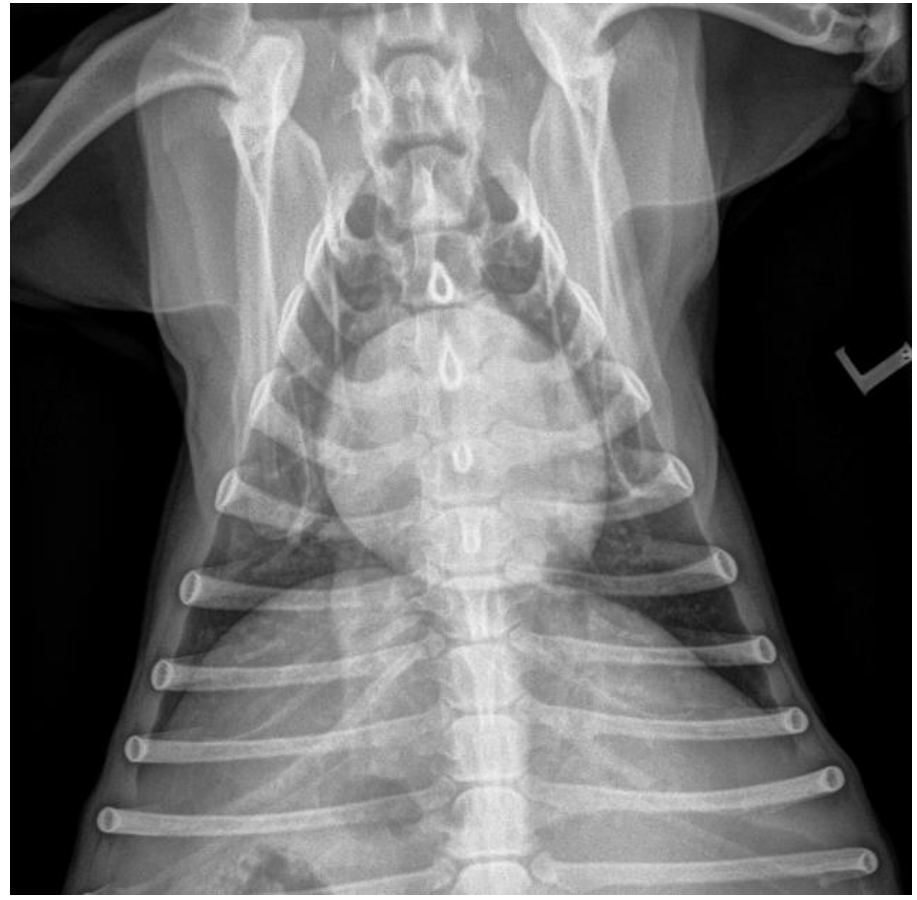

Figure 3. Thoracic radiograph, ventro-dorsal recumbency. (this tissue is less opaque than the soft tissue opacity of the displaced abdominal organs) although because of the size of the space-occupying fat opacity and having most of the abdominal organs pushed cranially, we cannot distinguish liver, spleen and kidneys properly. On the VD projection of the abdomen, multiple rounded soft tissue opacity nodules can be seen superimposed on the abdomen; these are consistent with the prominent nipples reported in the clinical findings, which are also seen superimposed on the ventral abdomen on the lateral radiograph.

\section{Key radiological findings are:}

- Reduced pulmonary field

- Fat opacity occupying most of the abdominal cavity, displacing the other organs cranially and dorsally. No discernible bladder shadow. 


\section{Differential diagnoses:}

Given the opacity of the mass lesion occupying the abdominal cavity, an adipose lesion is present, with the major differential being an intra-abdominal lipoma. Differential diagnoses could also include infiltrative lipoma or liposarcoma. The displacement of the abdominal organs and lack of evidence of generalised obesity indicates that a mass is present, as opposed to simply marked mesenteric fat deposition.

\section{What further investigations could you consider to try to obtain a diagnosis?}

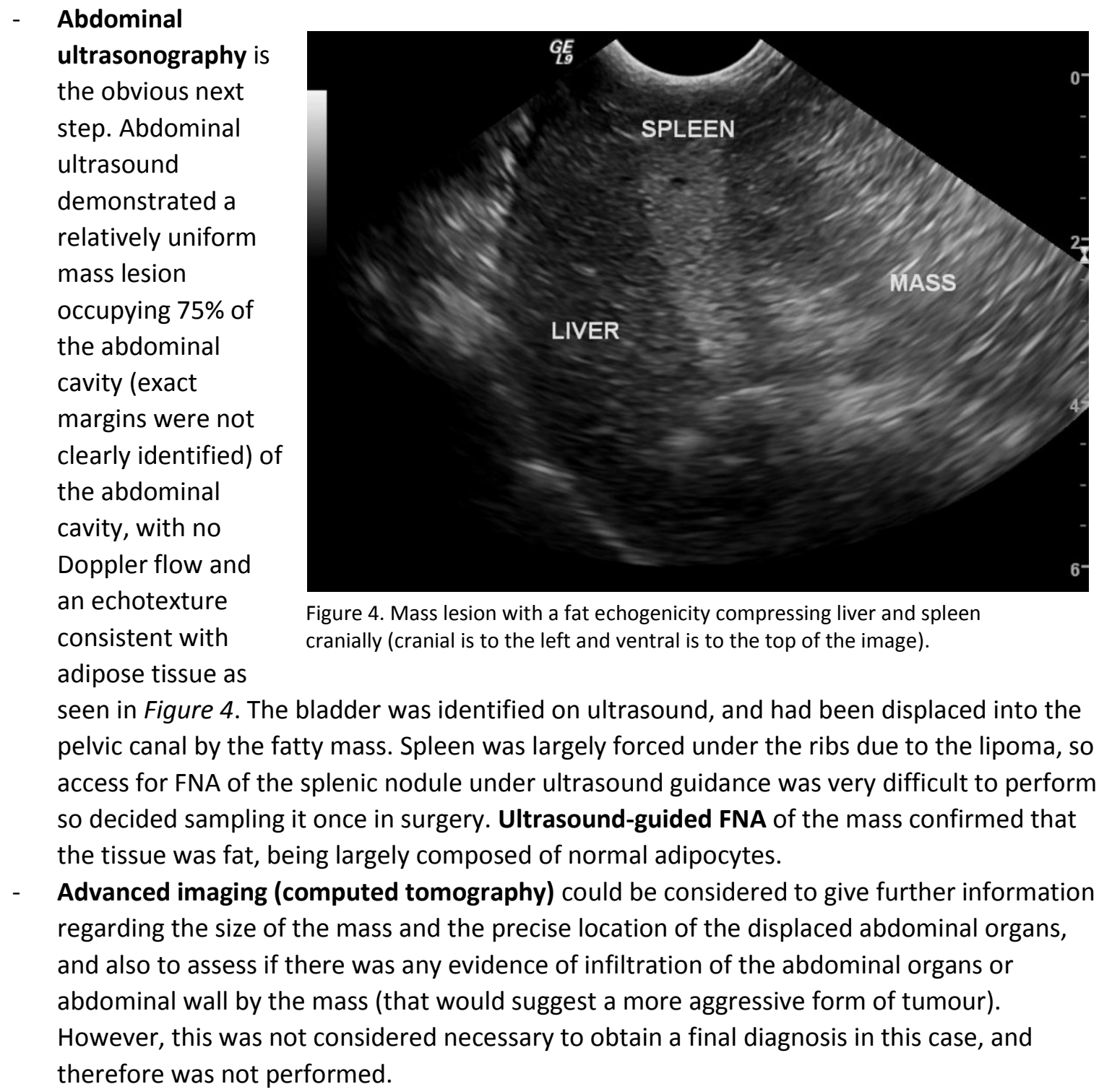

Figure 4. Mass lesion with a fat echogenicity compressing liver and spleen cranially (cranial is to the left and ventral is to the top of the image). adipose tissue as seen in Figure 4. The bladder was identified on ultrasound, and had been displaced into the pelvic canal by the fatty mass. Spleen was largely forced under the ribs due to the lipoma, so access for FNA of the splenic nodule under ultrasound guidance was very difficult to perform so decided sampling it once in surgery. Ultrasound-guided FNA of the mass confirmed that the tissue was fat, being largely composed of normal adipocytes.

- Advanced imaging (computed tomography) could be considered to give further information regarding the size of the mass and the precise location of the displaced abdominal organs, and also to assess if there was any evidence of infiltration of the abdominal organs or abdominal wall by the mass (that would suggest a more aggressive form of tumour). However, this was not considered necessary to obtain a final diagnosis in this case, and therefore was not performed. 
In addition to the fatty mass, abdominal ultrasound identified a $12 \mathrm{~mm}$ hypoechoic nodule in the spleen, as well as a hypoechoic mass in the right testicle (seen in Figure 5). Differentials for the testicular mass included neoplasia (Leydig cell tumour, Sertoli cell tumour). Differentials for the splenic nodule included haematoma, infarct, granuloma, regenerative

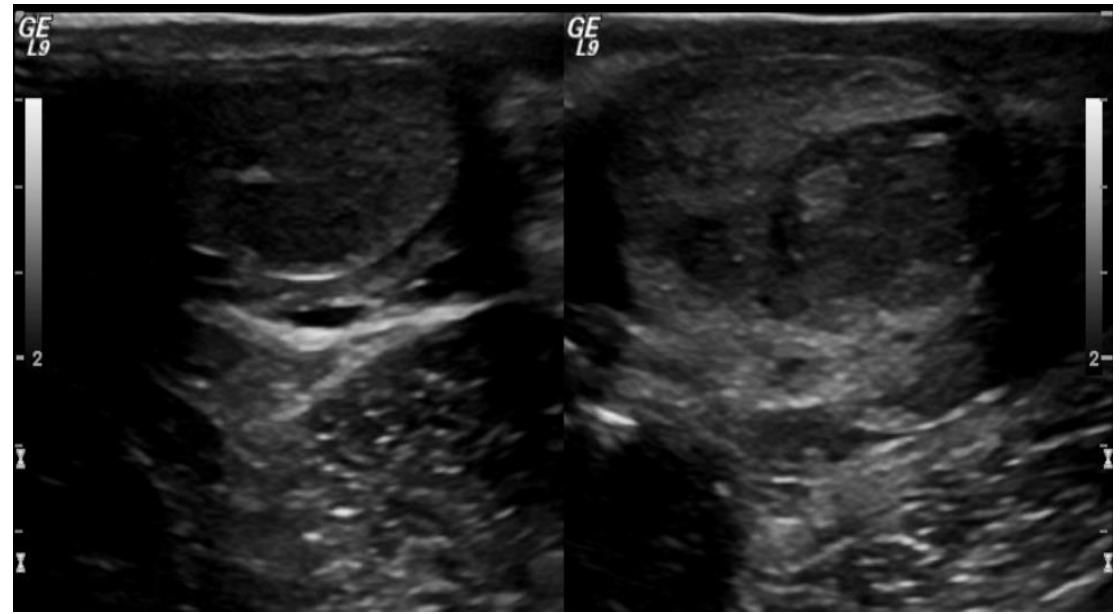

Figure 5. Ultrasonographic image comparing both testicles (left testicle on the left, right one on the right). Marked enlargement of the right testicle, as well as, the small hypoechoic mass within it. hyperplasia and neoplasia.

\section{Other investigations}

Due to the history of polydipsia, haematology, biochemistry and urinalysis were performed. Haematology and biochemistry revealed mild non-regenerative anaemia alongside a mild monocytosis; globulins were moderately elevated and albumin was moderately decreased. Otherwise blood results were unremarkable.

Urinalysis showed a specific gravity of 1.027 , marked proteinuria and haematuria. Urine culture demonstrated bacterial infection.

\section{Outcome}

It was concluded that the size of the abdominal lipoma was the cause of the majority of the clinical signs (largely through compression of the abdominal viscera), and the owners elected for exploratory laparotomy.

Once the dog was under anaesthesia, castration of the patient was decided as the right testicle contained a functional tumour pointing towards a feminisation syndrome. A sample of the testis was sent for histopathology.

Total resection of the abdominal tumour was performed and a $2.5 \mathrm{~kg}$ non infiltrative lipoma was removed. Additionally, the surgeon decided to carry out a partial splenectomy, removing the section containing the splenic nodule, and sent a representative sample for histopathological examination together with the sample from the neoplastic testicle.

The patient recovered well from the surgical procedure and was discharged that day with oral analgesia and prophylactic antibiotics (meloxicam (Metacam, Boehringer-Ingelheim) once daily for five days and amoxicillin-clavulanate (Synulox palatable tablets, Zoetis) $250 \mathrm{mg}$ twice a day for two weeks). No other follow-ups were made after that day in the hospital.

Histopathological examination of the splenic and testicular masses revealed a benign nodular hyperplasia of the spleen and two very common tumours within the abnormal testicle: an encapsulated interstitial (Leydig) cell tumour and a localised intraductal seminoma. Both tumours 
are low-grade neoplasms that usually remind localised to the testis, although occasionally may spread along the spermatic cord and rarely, metastasise to the regional lymph nodes. In this case, there was no evidence of involvement of the epididymis or pampiniform plexus, therefore castration was very likely to be curative (Borrego, 2017).

\section{Discussion}

Lipomas are very common in dogs, however their normal presentation is subcutaneous, with intracavity lipomas being much rarer. The breeds that seem to be particularly susceptible to the development of lipomas are the Labrador Retriever, Miniature Schnauzers and Doberman (Hammond and Regan, 2008)

The clinical signs caused by body cavity lipomas are those of a space-occupying lesion and depend on the body cavity involved, the growth rate of the tumour and the organs being compressed. These are slow-growing tumours, large in size and clinically silent until the animals show symptoms as a consequence of the compression of other organs or just because externally the condition becomes visible as the abdomen becomes distended. The dysuria and the UTI were most likely associated with the bladder compression by the mass, although this could not be proved.

In the specific case of intra-abdominal masses, abdominal distension is the first sign appearing and noticeable by the owner, after which, depending on the organ compressed, the following may develop: dysuria, tenesmus (if the mass has an intrapelvic extension and affects bladder/rectum), and digestive signs such as vomiting or diarrhoea, caused by compression of the organs in the cranial abdomen.

As mentioned above, recognising the type of opacity in radiographs is very important and can assist in the final diagnosis. Of 5 radiographic opacities that can be distinguished, fat opacity appeared to be present in this case. Fat, considered more radiolucent than soft tissue, absorbs relatively less radiation than bone or metal, so its images on the film will appear pale grey, producing radiographic contrast for differentiation and visualisation of the organs and structures. In this case, it exists variation in the opacity within the mass due largely to the thickness/amount of the fat centrally.

Another point to highlight here is the location of the mass. It has a central position in the abdominal cavity in this case. The difficulty in such cases is to determine the origin of the mass. It is important to pay attention to where the other organs have been displaced, because the direction of displacement provides useful information about the location of the mass (Barrett $E, 2013$ ). As shown in the images and reported earlier, the displacement of the majority of the organs in this case was cranial and dorsal, supporting the idea of a caudal origin of the mass, pushing the organs cranially and dorsally as the mass increased in size.

In general, and considering the different cases that have been reported in the literature on intracavity masses, the majority of the patients are old, and the results of the haematology and serum biochemical analyses are usually within the normal ranges, unless, as a result of the continuous compression of the organ for a long period of time, organ function (for example hepatic or splenic) has become compromised (Mayhew and Brockman, 2002).

Lipomas are normally not attached or do not have firm attachments to other structures (Klosterman et al, 2012; Mayhew and Brockman, 2002), although there is a report of an uncommon case of an 
intra-abdominal necrotic lipoma that was tightly adhered to the cranial aspect of the urinary bladder in an 11yo schnauzer (Hammond and Regan, 2008).

Ultrasonographically, intra-abdominal lipomas have a variable striated appearance, but most are uniformly hyperechoic, well-marginated, have a sharp edge definition (Mattoon and Nyland 2015) and a poorly vascularisation with some peripheral blood flow (Clapp et al, 2009).

In conclusion, all clinical signs related to the mass resolved after surgical resection of the mass (Klosterman et al, 2012; Mayhew and Brockman, 2002). Recurrence of the abdominal distension or grow of another abdominal mass are very rare with the exceptional case of a dog who had two intraabdominal lipomas 2 years apart, the second occurring after completely resection of the first mass (Mayhew and Brockman, 2002). The prognosis in these dogs is good, although 'new' or recurrent tumours may appear after the surgical procedure.

\section{References}

Clapp K, Rademacher N, Gaschen L (2009) Imaging diagnosis---Intra-abdominal lipoma Veterinary Radiology \& Ultrasound, Vol. 50, No. 6

Hammond TN, Regan J (2008) Imaging diagnosis-intra-abdominal necrotic lipoma Vet Radiol Ultrasound; 49:365-367

Klosterman ES, Gan Heng H, Freeman LJ, Childress M,O (2012) Transdiaphragmatic extension of a retroperitoneal lipoma into the intrathoracic extrapleural space via the lumbocostal trigone in a dog JAVMA, April 2015, Vol 240, No. 8

Kshirsagar AY, Nangare NR, Gupta V, Vekariya MA, Patankar R, Mahna A, Wader JV (2014) Multiple giant intra-abdominal lipomas: A rare presentation. Int J Surg April 18 5(7):399-402. doi: 10.1016/j.ijscr.2014.04.002

Mayhew PD, Brockman DJ (2002) Body cavity lipomas in six dogs. J Small Anim Pract. Apr; 43(4):17781

Miles J, Clarke D (2001) Intrathoracic lipoma in a Labrador retriever Journal of Small Animal Practice $42,26-28$

Morgan LW, Toal R, Siemering G, Gavin P (2007) Imaging diagnosis-Infiltrative lipoma causing spinal cord compression in a dog Veterinary Radiology \& Ultrasound, Vol. 48, No. 1, pp 35-37

Smereczyński A, Kołaczyk K, Bernatowicz E (2014) Intra-abdominal fat. Part III. Neoplasms lesions of the adipose tissue. J Ultrason. 2016 Jun; 16(65):145-54. doi: 10.15557/JoU.2016.0016. Epub 2016 Jun 29

Yang TW, Tsuei YW, Kao CC, Kuo WH, Chen YL, Lin YY (2017) Torsion of a Giant Antimesenteric Lipoma of the Ileum: A Rare Cause of Acute Abdominal Pain. Am J Case Rep. 2017 May 27; 18:589592 


\section{Further reading}

Barrett E - Radiology of the abdomen (2013). In Holloway JS and McConnell F BSAVA Manual of Canine and Feline Radiography and Radiology A Foundation Manual. British Small Animal Veterinary Association, Gloucester, UK. pp 191-193

Borrego, Juan F - Urogenital and Mammary Gland Tumours (2017). In Ettinger SJ, Feldman EC, Coté E Textbook of Veterinary Internal Medicine 'Volume 2' $8^{\text {th }}$ edition, Elsevier, Missouri USA. pp 2123

D'Anjou M-A, Carmel EN - Abdominal cavity, lymph nodes, and general great vessels (2015). In Pennick D and d'Anjou M-A Atlas of Small Animal Ultrasonography $2^{\text {nd }}$ edition, Wiley Blackwell, Oxford, UK. pp 467-468

Dennis R, Kirberger RM, Barr F, Wrigley RH - Other abdominal structures: abdominal wall, peritoneal and retroperitoneal cavities, parenchymal organs (2010). In Dennis R, Kirberger RM, Barr F, Wrigley RH Handbook of Small Animal Radiology and Ultrasound $2^{\text {nd }}$ edition, Saunders, Croydon, UK. pp 255262

Hayward, Nicolette - Abdominal masses; Baines Elizabeth A - The peritoneal cavity; Costello M - The male reproductive system (2012). In O'Brien Robert, Barr Frances BSAVA Manual of Canine and Feline Abdominal Imaging. British Small Animal Veterinary Association, Gloucester, UK. pp 35-36, 76$86,243-244$

Widmer, William R, Mattoon, John S, and Nyland, Thomas G - Peritoneal Fluid, Lymph Nodes, Masses, Peritoneal Cavity, Great Vessel Thrombosis, and Focused Examinations (2015). In Mattoon, John S, Nyland, Thomas G Small Animal Diagnostic Ultrasound $3^{\text {d }}$ edition, Elsevier, Missouri USA. pp 501-16 